\title{
Endoplasmic reticulum: a focal point of Zika virus infection
}

\author{
Muhammad Izzuddin Mohd Ropidi, Ahmad Suhail Khazali, Nurshamimi Nor Rashid ${ }^{*}$ (D) and Rohana Yusof
}

\begin{abstract}
Zika virus (ZIKV) belongs to the Flavivirus genus of the Flaviviridae family. It is an arbovirus that can cause congenital abnormalities and is sexually transmissible. A series of outbreaks accompanied by unexpected severe clinical complications have captured medical attention to further characterize the clinical features of congenital ZIKV syndrome and its underlying pathophysiological mechanisms. Endoplasmic reticulum (ER) and ER-related proteins are essential in ZIKV genome replication. This review highlights the subcellular localization of ZIKV to the ER and ZIKV modulation on the architecture of the ER. This review also discusses ZIKV interaction with ER proteins such as signal peptidase complex subunit 1 (SPCS1), ER membrane complex (EMC) subunits, and ER translocon for viral replication. Furthermore, the review covers several important resulting effects of ZIKV infection to the ER and cellular processes including ER stress, reticulophagy, and paraptosis-like death. Pharmacological targeting of ZIKVaffected ER-resident proteins and ER-associated components demonstrate promising signs of combating ZIKV infection and rescuing host organisms from severe neurologic sequelae.
\end{abstract}

Keywords: Zika virus, Endoplasmic reticulum, Unfolded protein response, Stress granules, Reticulophagy, Paraptosis, Cytoplasmic vacuolization

\section{Introduction}

Zika virus (ZIKV) is a mosquito-borne virus that belongs to the Flaviviridae family together with other notable flaviviruses such as dengue virus (DENV), West Nile virus (WNV), Japanese Encephalitis virus (JEV), and yellow fever virus (YFV). ZIKV was first isolated from a febrile rhesus monkey in April 1947 and was subsequently isolated from Aedes africanus mosquitoes 9 months later in Zika Forest of Uganda [1] \{Dick, 1952 $\# 15\}$. Despite its discovery more than a half-century ago, ZIKV received little attention due to sporadic cases of human infection with mild and self-limiting symptoms [2]. ZIKV was put under scrutiny following its first outbreak in the Yap Island of Micronesia in 2007. During this outbreak, 185 suspected cases of ZIKV infection were reported, and at least $24 \%$ of these patients were either serologically or molecularly confirmed for ZIKV infection [3]. The same study estimated that 5005 island residents (73\%) were infected, of which 919 were symptomatic [3].

\footnotetext{
* Correspondence: nurshamimi@um.edu.my

Department of Molecular Medicine, Faculty of Medicine, University of Malaya, 50603 Kuala Lumpur, Malaysia
}

ZIKV-infected patients typically present mild clinical symptoms such as fever, maculopapular rash, conjunctivitis, and arthralgia [2]. Nevertheless, the second ZIKV outbreak in French Polynesia in 2013 provided the first compelling relationship between ZIKV infection and a neurological complication, where a woman was diagnosed with Guillain-Barré syndrome (GBS), an autoimmune disease typically affecting motor neuron functions, a week following the onset of ZIKV-like symptoms [4]. This epidemic also recorded a 20-fold increase of GBS incidence, where 41 GBS-diagnosed patients (98\%) were serologically positive for ZIKV [2, 5].

The sudden spike of microcephaly cases among newborns in Brazil following the 2015-2016 ZIKV outbreak triggered the Brazilian Ministry of Health and the World Health Organization to declare ZIKV as a national and international public health emergency $[6,7]$. The causative link between ZIKV and congenital neurological anomalies was established based on several evidence including the detection of ZIKV RNA within the amniotic fluid acquired from ZIKV-infected mothers with confirmed fetal microcephaly case $[8,9]$. Subsequent clinical and pre-clinical findings further supported the causal 
relationship between ZIKV infection and microcephaly in newborns [10-12].

ZIKV is believed to infect cells through receptormediated endocytosis. These putative receptors include Cluster of Differentiation 209 (CD209), Tyrosine-protein kinase receptor Tyro3, and AXL, where overexpression of these receptors in ZIKV-impervious HEK293T cells rendered the cells susceptible to ZIKV infection [13]. In particular, the role of AXL in ZIKV infection has been extensively investigated due to its crucial role in dengue virus infection [13]. During ZIKV infection, AXL mediates ZIKV entry indirectly whereby the phosphatidylserine extension on ZIKV lipid membrane binds to Growth arrest-specific 6 (Gas6), one of the ligands for AXL that serves as a bridge for ZIKV and AXL interaction, resulting in clathrin-mediated virus internalization [14, 15]. The acidic microenvironment within the endosome promotes fusion between the virus envelope proteins and the endosomal membrane resulting in the release of ZIKV genome into the host cell cytosolic space $[15,16]$.

The ZIKV genome is a positive-sense, single-stranded RNA ((+)ssRNA) of approximately 11,000 bases in length [2]. ZIKV genome contains a single open reading frame that encodes three structural and seven non-structural (NS) proteins. These structural proteins consist of capsid (C), pre-membrane (prM), and envelope (Env) proteins, which are predominantly involved in viral pathogenesis and virion structure. The seven non-structural proteins, NS1, NS2A, NS2B, NS3, NS4A, NS4B, and NS5 proteins, largely contribute towards the purposes of viral pathogenesis, replication, and immune evasion [17]. ZIKV utilizes host translational machinery to produce a single polyprotein that is further cleaved by viral NS2B-NS3 serine protease and host cell protease into functional viral proteins [18]. These viral proteins are then distributed to cellular compartments for various functions [19].

\section{ZIKV proteins localize to distinct subcellular compartments}

ZIKV proteins are primarily distributed within and in close proximity to several endomembrane compartments including the endoplasmic reticulum, Golgi apparatus, endosomes, lysosomes, autophagosomes, and nucleus [20]. Molecular cloning and expression of individual ZIKV proteins reveal distinct and specific organellar localization. ZIKV capsid localizes to several compartments including the nucleus and the Golgi [19]. In addition to capsid, NS2B and NS4A also localize to the Golgi. Three ZIKV proteins namely Env, prM, and NS2A are distributed to the ER as indicated by their co-localization with calreticulin expression. NS5, which contains nuclear localization signals, forms punctate distribution in the nucleus [19]. However, these data should be interpreted with caution as the subcellular localization of these cloned viral proteins may differ in ZIKV-infected cells. For example, individual expression of ZIKV NS3 localizes to the mitochondria, but NS3 is instead localized at the ER when co-expressed with NS2B [21]. Similarly, although NS5 protein is primarily detected in the nucleus of ZIKV-infected cells [22], ZIKV NS5-RNA polymerase has been shown to interact with ZIKV NS3-helicase during viral RNA replication in the ER [23]. Inhibiting this interaction reduces NS3helicase activity. Subcellular localization of viral proteins is affected by viral proteins interaction, which is crucial for productive infection. Importantly, understanding the mechanisms of viral protein transport and viral protein-protein interaction may provide novel therapeutic targets.

The subcellular distribution of certain ZIKV proteins was corroborated in a separate study where interatomic analyses using proximity-dependent biotin-identification (BioID) labeling and FLAG-based immunoprecipitation (IP) coupled with mass spectrometry (MS) uncover indepth molecular interactions between ZIKV proteins with various host organelles and proteins. In general, ZIKV proteins mainly interact with host proteins that are involved in protein processing, vesicle trafficking, RNA processing, and lipid metabolism [20]. ZIKV capsid interacts with multiple nucleolar proteins, whereas NS5 protein targets and disrupts the Cajal bodies in the nucleus [20]. This observation is consistent with the aforementioned experimental study that showed nuclear localization of ZIKV capsid and NS5 protein [19]. The study also identified numerous interactions between viral proteins (prM, Env, NS2A, NS2B, NS4A, and NS4B) with various ER proteins, which highlights the importance of the ER in ZIKV life cycle [20].

ZIKV has been reported to be dependent on several ER proteins including ER-associated signal peptidase complex (SPC) proteins, ER translocon, and ER membrane complex (EMC) proteins. SPC, especially SPC subunit 1 (SPCS1), is crucial for ZIKV pathogenesis as knocking out SPCS1 in $293 \mathrm{~T}$ cells significantly reduced ZIKV infection and drastically impaired the production of infectious ZIKV particles [24]. A study using pooled CRISPR/Cas9 cell survival enrichment assay identified ZIKV strongly depends on ER membrane complex (EMC) during early-stage ZIKV replication [25]. EMC is a highly conserved oligomeric complex residing on the ER membrane and is crucial for transmembrane protein folding and lipid trafficking [25]. The dependency on six EMC subunits (EMC1-EMC6) was verified with siRNA assays, where depletion of these proteins significantly impaired the replication of several ZIKV strains [25]. Another study reported that ZIKV NS4B physically interact with EMC1 subunit and depletion of this subunit markedly reduced the level of ZIKV NS4A and NS4B protein [26], indicating that EMC proteins are 
required for viral protein biogenesis. Further investigation using DENV NS4B identified two marginally hydrophobic domains at the N-terminal of NS4B to be crucial for NS4B dependence on EMC [26]. Since ZIKV NS4B shares moderate sequence identity, high sequence similarity, and similar topology with other Flaviviruses [27], it is plausible that the two weak hydrophobic domains of ZIKV NS4B are the specific determinants for ZIKV dependency on EMC proteins. In addition to its direct interaction with viral proteins, EMC proteins also associate with ER Sec61 translocon and oligosaccharyltransferase (OST) complex proteins, both of which are also important for ZIKV infection [24, 25]. Sec61 is a major component of the ER translocon that facilitates the entry of nascent polypeptides into the ER lumen for protein processing. ZIKV dependency on Sec61 translocon was validated in a separate study wherein myolactone treatment, a Sec61 $\alpha$ inhibitor, dramatically reduced ZIKV expression [28]. ZIKV replication was restored in cells expressing mutant Sec61 $\alpha$ that conferred resistance against myolactone inhibition [28]. ZIKV dependency on OST complex, an integral part of the translocon consisting of eight ER-transmembrane protein subunits that catalyzes co-translational $\mathrm{N}$-glycosylation, is based on EMC1, EMC2, EMC4 and EMC5 interaction with OST complex subunits namely STT3A/B, RPN1/2, and DDOST [25]. Additionally, ZIKV proteins including NS4B have been reported to directly interact with these OST subunits [29]. ZIKV dependency on OST complex is corroborated by Marceau et al. that reported a significant abrogation of ZIKV RNA replication in STT3A knockout cells [30]. Importantly, treatment with NGI-1, a small molecule OST complex inhibitor, significantly reduced ZIKV RNA replication with an $\mathrm{EC}_{50}$ value of $2.2 \mu \mathrm{M}$ [31]. The inhibitory activity of this non-toxic molecule $\left(\mathrm{CC}_{50}=\right.$ $34.9 \mu \mathrm{M})$ is independent of $\mathrm{N}$-linked glycosylation activity of the OST complex as ZIKV replication was drastically impaired in HAP1 cells containing wild-type or catalyticinactive STT3A [31]. The specific mechanism of ZIKV dependency on STT3A is unclear, but immunoprecipitation and electron microscopy studies showed physical interactions between DENV proteins and STT3A/B, forming viral RNA replication complexes that reside within the ER in close proximity to DENV2-vesicle packets [30]. Since ZIKV proteins also directly interact with STT3A and ZIKV infection induces the formation of vesicle packets $[29,32]$, it is plausible that ZIKV depends on OST complex in a similar manner.

In short, ZIKV proteins interact with various host proteins and exploit their functions to accommodate the progression of the viral life cycle. ER and ER-related proteins are especially important in the virus genome replication, which may be useful in developing antiviral therapies. The following sections of this review will discuss the structural and molecular changes in the ER following ZIKV infection.

\section{ZIKV modulates ER structure}

Throughout the course of infection, viruses induce various modifications on the host cells' metabolisms and their cytoarchitecture to support the progression of virus life cycle. One of the common targets is the ER [33]. Among the important functions of the ER include protein processing, synthesis of essential biosynthetic compounds (primarily lipids and proteins), metabolism of steroids and carbohydrates, and storage of calcium ions $\left(\mathrm{Ca}^{2+}\right)$ for intracellular signaling [34]. Structurally, the ER is made of a continuous network of membraneenclosed flattened sacs and tubules. The dynamic and fluid nature of these structures enable this organelle to accomplish tubule extension, membrane curvature and shape alteration in response to stress-induced or factordemanding conditions such as cell division and differentiation [34]. Likewise, ZIKV infection is also capable of exploiting the organelle's plasticity and remodels the ER structure for their replicative benefit.

Recent transmission electron microscopy analyses on ZIKV-infected cells frequently observed significant expansion of the ER [32, 35, 36]. This structural modification typically consisted of proliferating ER lamellae and dilated ER lumen, which cumulatively increases the overall ER size and volume. Enlargement of the ER is believed to occur in response to virus-induced ER stress, due to a supply-demand imbalance that will be elaborated in the next section.

ZIKV infection also forms distinct aggregates of intricate ER tubular network that resembles sponge-like matrix called the convoluted membranes (CM) (Fig. 1) [32, 35-37]. CM aggregates are frequently seen bordering ZIKV virions and other ZIKV-induced structures, with occasional virions also found within the $\mathrm{CM}$ itself $[32,36]$. The function of the CM in ZIKV infection is unclear; nevertheless, evidence from other flaviviruses studies suggest that the CM possesses roles closely associated to protein synthesis and processing [18]. Interestingly, CM aggregates are not observed in ZIKV-infected neural progenitor cells, suggesting cell-type-specific factors are required to develop the CM [32]. No discernible difference in the CM structure is observed between the African and Asian ZIKV strains [36, 37].

Next, vesicle packets (VPs) are also commonly observed in ZIKV-infected cells (Fig. 1) [32, 35-37]. VPs refers to an aggregate of closely packed single-membrane vesicles that are encapsulated within an ER cisterna. Individually, these vesicles measure between 60 and $100 \mathrm{~nm}$ in diameter with a narrow channel of approximately $10 \mathrm{~nm}$ that connects the vesicle lumen and the cytosol $[32,35]$. The radial dimension of these vesicles varies according to the host cell type, thereby hinting possible involvement of cell-type-specific factors in its formation [36]. Modest radial difference was also noted between ZIKV lineages [32]; 

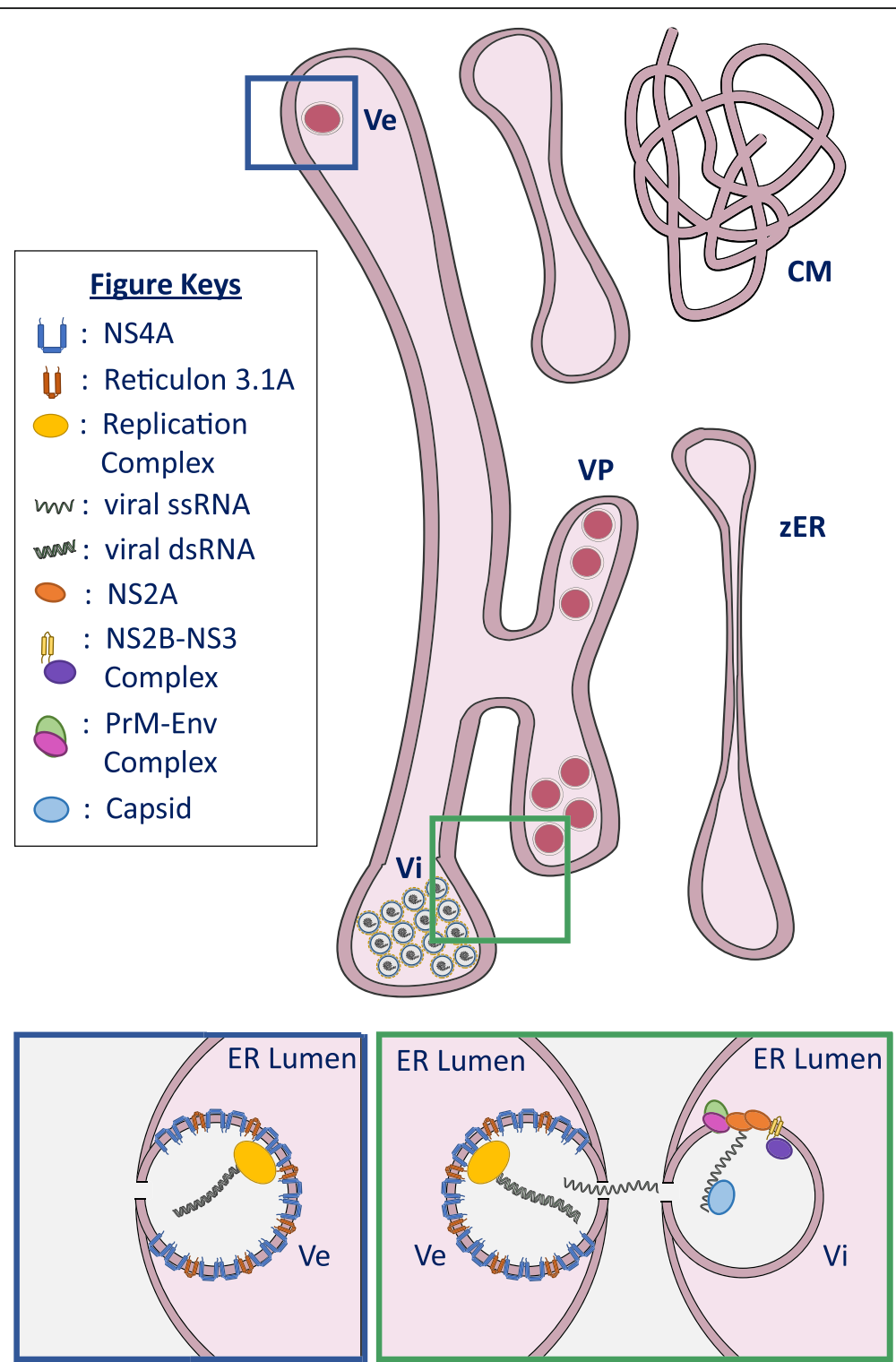

Vesicle Invagination Viral RNA Release and Virion Assembly

Fig. 1 ZIKV infection induces remodeling of the ER structure. ZIKV exploits the ER dynamic characteristic and remodels the ER structure to generate virus-induced structures, vesicles (Ve), vesicle packet (VP), convoluted membrane (CM) and zippered ER (zER), for the benefit of virus replication. Blue and green box depicts schematic representation of host and viral factors involved in vesicle and virus particle (Vi) formation, respectively. ZIKV NS4A utilizes the host reticulon 3.1A (RTN3.1A) to facilitate membrane curvature during vesicle invagination into the ER lumen (blue box). Viral genome replication takes place within this vesicle. Neosynthesized viral RNA genome is released into the cytosol and could undergo either translation, virus particle assembly, or another round of genome replication. Virus particle assembly takes place in apposed ER leaflet. Separate ZIKV NS2A independently recruits viral genome RNA, NS2B-NS3, and unprocessed C-prM-Env complexes, and subsequently congregates at the virus particle assembly site through NS2A oligomerization (green box). Once assembled, NS2B-NS3 proteolytical activity cleaves the recruited C-prM-Env complex to generate individual capsid, prM and Env protein. Cleaved capsid protein interacts with capsid-dense lipid droplets and viral RNA to generate nucleocapsid core followed by Env and prM proteins encapsulation of the nucleocapsid core

however, more morphologically pronounced difference between the ZIKV lineages is observed in the vesicle's shape whereby African strain tend to form ovoid vesicles, whereas the Asian strain's vesicles are generally more spherical [37]. Nevertheless, these differences, especially the latter, remain contentious due to limited experimental measurements and warrants additional investigations across various cell types to support these observations. Despite these differences, the vesicles' pore maintain a comparable diameter of $10 \mathrm{~nm}$ regardless of the virus strain and the host cell type, suggesting that a conserved cellular or viral machinery mediates pore formation [32]. 
Invagination of ZIKV-induced vesicles into the ER lumen is mediated through the host reticulon 3.1A (RTN 3.1A) that facilitates ER membrane curvature (Fig. 1, blue box) [38]. Silencing of RTN3.1A impairs NS4A protein stability and results in significant reduction of virusinduced vesicles and virus replication [38]. Typically, these vesicles contain the virus' replication complex and therefore carries out the virus RNA synthesis or viral replication $[32,36]$. Following viral replication, neosynthesized viral genome RNA is released into the cytosol through the vesicle's narrow channel, where it would consequently either undergo another round of genome replication, translation or virion assembly $[17,30]$. In the latter case, ZIKV virion assembles and buds into the ER lumen directly apposed the narrow pore of the replication vesicle (Fig. 1, green box) [32].

Although detailed mechanistic insights of virion assembly remains uncertain, recent study identified that ZIKV NS2A independently recruits viral (+)-ssRNA, NS2B-NS3, and unprocessed C-prM-Env complexes to the virus particle assembly site, possibly through NS2A oligomerization [39]. The same study postulates that virus particle assembly proceeds through NS2B-NS3 protease complex cleaving the recruited C-prM-Env complex. Cleaved capsid protein form nucleocapsid core with the viral RNA and capsid-dense lipid droplet; and subsequently, it is encapsulated by prM and Env proteins, which leads to eventual virion budding into the ER lumen [39]. Ultrastructural analyses within the dilated ER lumen consistently identified virus particles arranged in a two-dimensional paracrystalline array [32, 37]. This array is located in close proximity to the replication vesicles and is therefore largely comprised of fully assembled virions and viral-like particles (enveloped virions without viral genome) [37].

Moreover, ZIKV-infected Huh7 cells also exhibited a unique structural alteration of collapsed ER cisterna or more commonly known as zippered ER (zER) (Fig. 1) that reminisce the observation reported in avian infectious bronchitis virus (IBV) infection, a (+)-ssRNA virus from the Coronaviridae family $[32,40]$. This finding presents the first identification of such structure among flavivirus-infected cells and has only been observed in ZIKV-infected Huh7 cells, suggesting that cell-typespecific factors are possibly required to generate this structure [32]. The role of zER in ZIKV infection is unclear; however, zER are frequently found adjacent to VPs suggestive of functions associated with vesicles formation, which is consistent with observations made in IBVinfected cells [32, 40]. Nonetheless, this unique finding warrants further research to elucidate the mechanistic details and biological relevance of the zER.

In addition to the direct modulation of the ER structure, ZIKV also induces the formation of viroplasm-like structures, which is incidentally the first reported observation of such structure in flavivirus infection [41]. Contrary to other ZIKV-induced structures, viroplasms are cytoplasmic inclusion of viral replication components and various relevant host factors associated with virus replication that are formed through reorganization of cell membrane or cytoskeletal elements [42]. These structures are significantly larger than VPs and can measure up to $500 \mathrm{~nm}$ in diameter [41]. Viroplasms are primarily located in the perinuclear region and in close proximity to the ER, mitochondria, and microtubules to facilitate viral genome replication [41].

The spatial segregation of virus-induced enclosed structures (viroplasms and vesicles) is postulated to create a subcellular microenvironment concentrated with factors required for virus genome replication, and simultaneously provides physical barriers against RNA nucleases and the host immune responses [32, 42]. The latter, in particular, is consistent with an investigation on WNV that virusinduced structures confer protection, albeit partially, against the host immune mechanism [43].

In summary, ZIKV and several ZIKV proteins localize to the ER and eventually leads to the remodeling of the reticular architecture and exploitation of the organelle's unique characteristics. These modifications create an ideal environment for viral genome replication, but simultaneously introduce additional burden and stress on the host cell leading to the activation of several cellular responses, which will be discussed in the following sections.

\section{ZIKV induces ER stress and the unfolded protein response}

One of the fundamental roles of the ER is the folding of secreted and membrane proteins, which depends on a multitude of factors including supply of ATPs, stable $\mathrm{Ca}^{2+}$ concentration, and a balanced redox environment [44]. Disruption of this specialized environment within the ER, whether through glucose deprivation or viral infection, may lead to overwhelming accumulation of misfolded and unfolded proteins - a condition described as ER stress [45].

Under normal conditions, glucose regulated protein 78 (GRP78), one of the ER molecular chaperones, binds to ER stress sensor transmembrane proteins: protein kinase RNA-activated (PKR)-like ER resident kinase (PERK), activating transcription factor 6 (ATF6), and inositolrequiring enzyme 1 (IRE1). In the presence of misfolded or unfolded protein, GRP78 will bind to exposed hydrophobic residues of these proteins to induce proper protein folding $[45,46]$. Accordingly, during ER stress, the accumulation of misfolded or unfolded proteins saturates the free pool of GRP78 and titrates the bound GRP78 away from the stress sensors leading to activation of respective sensor's molecular pathways [45]. 
These molecular initiatives and their corresponding downstream effects are collectively known as the unfolded protein response (UPR). This evolutionarily conserved countermeasure induces pro-survival responses including global arrest of protein synthesis, upregulation of protein degradation factors, and enhanced protein folding capability [45]. However, under severe ER stress conditions, UPR will instead trigger apoptosis.

Following ZIKV infection, the accumulation of misfolded virus polyproteins in the ER lumen overwhelms the ER protein-folding capacity leading to ER stress and triggers the activation of the UPR (Fig. 2) [47]. Additional evidence of ER stress and UPR activation are demonstrated in the elevated expression of GRP78 and other chaperones such as calnexin, calreticulin, and protein disulfide isomerase (PDI) in ZIKV-infected neural cells in vitro and in vivo $[35,48,49]$. Increased expression of these ER stress markers was accompanied by impaired indirect neurogenesis and microcephalic phenotype in mice [49]. This finding is consistent with a previous report wherein the induction of UPR in cerebral apical progenitor cells tipped neuronal differentiation towards direct neurogenesis at the expense of indirect neurogenesis, leading to depleted intermediate progenitors, reduced overall cortical neuron output, and diminished cerebral volume, which ultimately caused microcephaly in vivo [50].

In addition to elevating the expression of ER chaperone proteins, ZIKV has been shown to induce UPR by activating the stress sensors as summarized below (Fig. 2).

\section{ZIKV and ATF6}

ATF6 is a type II ER transmembrane protein that contains a transcription activation domain (TAD) on its cytosolic-facing N-terminal and two independent Golgilocalization signals (GLS) on its luminal-facing Cterminal [51]. Following the dissociation of GRP78 that unmasks the GLS, ATF6 translocate to the Golgi apparatus and undergoes sequential proteolytic processing by

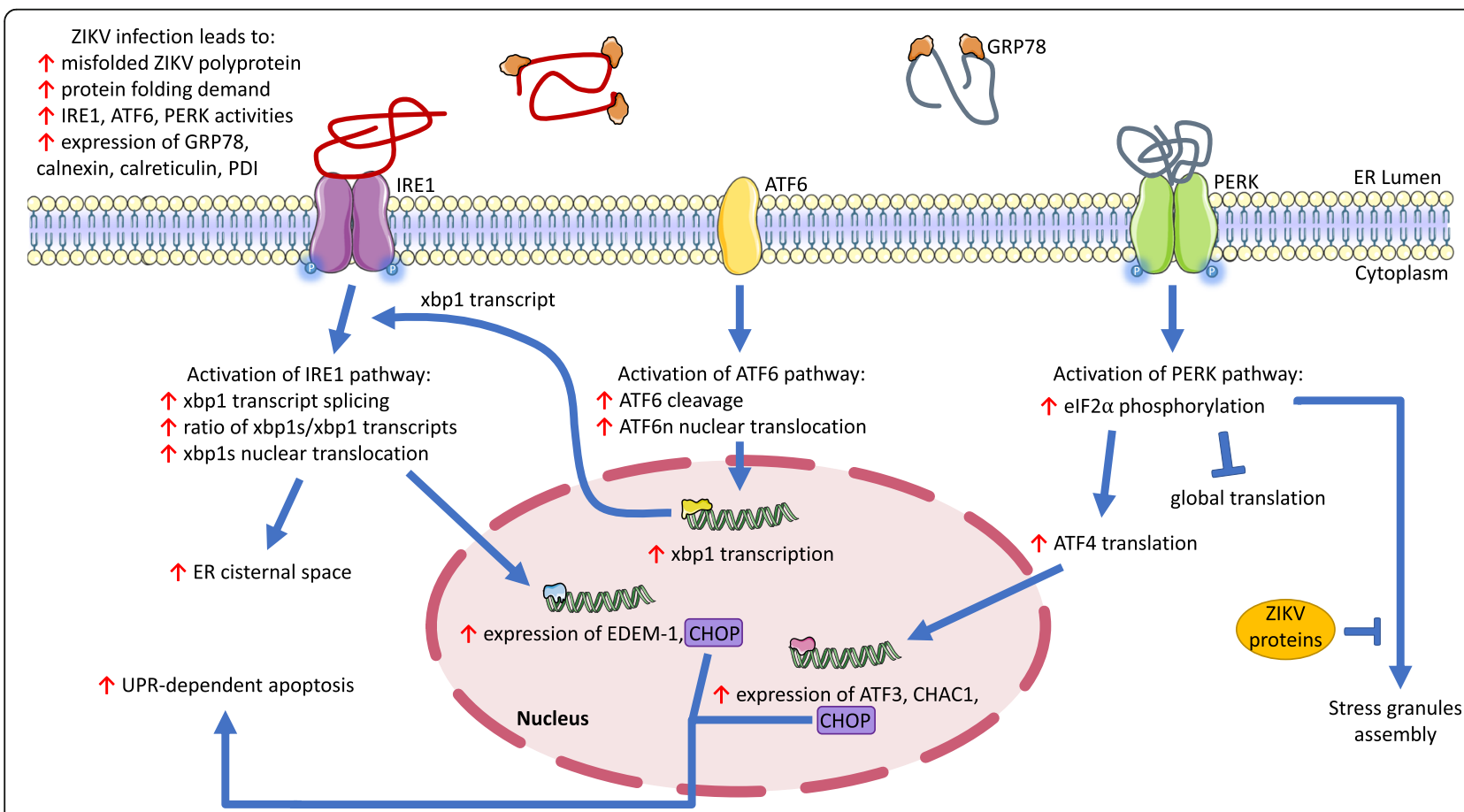

Fig. 2 ZIKV-induced ER stress initiates host cell unfolded protein response (UPR). ZIKV infection induces ER stress due to the increased amount of unfolded/misfolded viral (red strand) and host cell (grey strand) protein aggregates in the ER lumen. The accumulation of these partially processed proteins leads to the overwhelming demand of correct protein folding. To facilitate protein folding, GRP78 (orange) dissociates from the stress sensors (IRE1, ATF6, and PERK) and binds to the misfolded/unfolded proteins. Expression of other chaperones is also elevated to address the overwhelming protein folding demand during ZIKV infection. Binding of misfolded protein to PERK (green) activates the sensor and triggers phosphorylation of elF2 that in turn stimulates 1) global translational block except for selective mRNA involved in UPR, and 2) formation of stress granules. However, ZIKV bypasses global translation block and inhibits stress granules formation, but the mechanistic details of these viral interference are still unclear. Activation of ATF6 (yellow) promotes the protein proteolytical processing in the Golgi apparatus into ATF6n, ATF6n nuclear translocation, and expression of UPR target genes including XBP1. Activated IRE1 (purple) splices XBP1 transcript which produces an active transcription factor (XBP1s) that stimulates expansion of the ER volume, and expression of CHOP and ERAD factors such as EDEM-1. Alternatively, IRE1 can trigger ASK1-p38 MAPK pathway that enhances CHOP apoptotic activities and promotes other apoptotic-related activities under severe ER stress condition. Blue pointed arrows denote activation, blue blunt-end arrows denote inhibition, and red pointed arrows denote increased expression/activity 
site- 1 and site- 2 proteases, liberating TAD-containing ATF6 N-terminal (ATF6n) [51]. Subsequently, ATF6n enters the nucleus and binds to ER stress response elements in the promoter region of UPR-target genes, including X-box binding protein 1 (XBP1) transcription factor, a downstream effector of IRE1 stress sensor pathway, and GRP78 to autoregulate the UPR [51-53].

ZIKV infection has been shown to activate the UPR via ATF6, where ATF6 proteolysis and ATF6n nuclear translocation were induced in vitro and in vivo [48].

\section{ZIKV and IRE1}

IRE1 is a single-pass transmembrane protein. The protein's ER luminal domain serves as a stress sensor, whereas the cytosolic domain is sub-divided into kinase and ribonuclease (RNase) domains. Under ER stress, GRP78 releases from IRE1 luminal domain and binds onto the misfolded/unfolded proteins. This dissociative event frees the luminal domain and in turn, permits binding of the misfolded/unfolded protein onto the liberated domain $[54,55]$. Consequently, IRE1 oligomerizes at its luminal domain, which brings the kinase domains in close proximity leading to auto-phosphorylation of the kinase domains [56]. The phosphorylation event at the activation loop is postulated to induce conformational changes of the RNase domain that permit a more efficient binding of RNA substrates [57]. Activated IRE1 cleaves off 26 nucleotides from XBP1 transcripts, resulting in a translational frameshift to produce a potent transcriptional activator (XBP1s) that elevates the expression of UPR-target genes to promote protein folding, processing, and secretion $[53,58]$. XBP1s also modulates the expression of factors involved in ER-associated protein degradation (ERAD), a cellular process of eliminating aberrant proteins through retro-translocation and proteasomal degradation [45].

Alternatively, IRE1 protein can also mediate ER stressinduced apoptosis following chronic stress damage by triggering activation of apoptotic signaling kinase 1 (ASK1), which consequently activates p38 mitogen-activated protein kinase (p38 MAPK) and Jun N-terminal kinase (JNK) leading to apoptosis [59]. JNK mediates apoptosis primarily by inhibiting the anti-apoptotic B-cell lymphoma 2 (BCL-2) and activating the pro-apoptotic BIM protein [59], whereas p38 MAPK promotes apoptosis by phosphorylating CCAAT-enhancer-binding protein (C/EBP) homologous protein $(\mathrm{CHOP})$ at serine residues 78 and 81 to enhance its transcriptional activity and induce apoptosis $[60,61]$. CHOP is expressed downstream of all three ER stress sensors, indicating overlapping UPR pathways during severe ER stress [62]. CHOP forms heterodimers with other C/EBP family transcription factors; therefore, the basic domain in CHOP renders the transcription factors incapable of binding to their respective DNA binding sites, which reduces the expression of their target proteins including BCL-2 [63]. CHOP also contains a TAD and thus, can bind to a unique sequence to induce the expression of target genes including BIM [63, 64].

ZIKV infection has been shown to promote XBP1 splicing and $\mathrm{XBP} 1 \mathrm{~s}$ translocation into the nucleus, leading to elevated expression of XBP1 downstream effectors such as ER degradation-enhancing $\alpha$-mannosidase-like 1 (EDEM1 ) and CHOP, indicating the activation of the IRE1 arm of the UPR [48]. EDEM-1 is an enzyme involved in the ERAD process that recognizes and facilitates retrotranslocation of misfolded proteins to the cytosol for subsequent proteasomal degradation and thus, alleviating stress damage [65]. Instances of aggravated ER stress were also demonstrated in ZIKV-infected cells, which lead to elevated expression of $\mathrm{CHOP}$ protein and initiation of ERstress-induced apoptosis [49]. Pharmacological intervention using IRE1 inhibitor, $4 \mu 8 \mathrm{C}$, prevented microcephaly and impaired ZIKV replication in mouse fetal brains [49]. Modulation of IRE1-ASK1 pathway during ZIKV infection is still unclear.

Previous study reported that ectopic expression of XBP1 promotes expansion of the ER, a visual sign often manifested in response to ER stress [66]. This morphological change is hypothesized to reduce the local concentration of misfolded/unfolded proteins and accommodate the overwhelming demand for protein folding-a significant portion of which is neosynthesized viral polyproteins $[47,67]$. Consistent with increased expression of XBP1, ultrastructural characterization of ZIKV-infected cells reported significant ER enlargement extending across the cytoplasm and is related to ZIKV-induced ER stress [32, 35, 36, 47].

\section{ZIKV and PERK}

Similar to IRE1, release of GRP78 proteins enable misfolded/unfolded proteins binding onto exposed PERK luminal domain leading to oligomerization and autophosphorylation of PERK protein to activate its downstream pathway [55, 68]. PERK phosphorylation, in turn, induces eIF $2 \alpha$ phosphorylation (p-eIF $2 \alpha$ ) that mediates three interlinked UPR mechanisms: expansion of protein folding capacity, suppression of nascent protein production and induction of apoptosis in the event of severe stress [69]. Phosphorylation of $\alpha$ subunit in the eIF2 complex hinders the assembly of preinitiation complex (PIC) by blocking the activity of eIF2B guanine exchange factor, leading to global suppression of mRNA translation [69]. However, selected mRNAs, typically transcripts for UPR machinery, can bypass this translational block [69]. Interestingly, viruses have evolved various mechanisms to overcome this translational block, but the mechanism of this process in ZIKV infection is unclear. Recent analyses of ZIKV infection in vitro and in vivo reported a 
substantial increase of eIF $2 \alpha$ phosphorylation and elevated expression of several downstream PERK effectors such as ATF4, ATF3, CHAC1, and CHOP [48, 49]. Importantly, intracerebroventricular administration of pharmacological PERK inhibitor in ZIKV-infected mice restored appropriate neurogenesis balance and rescued infected mouse embryos from microcephalic phenotype. However, unlike IRE1 inhibitor, PERK inhibitor did not affect ZIKV replication [49]. PERK inhibitor also prevented microcephaly in placental ZIKV inoculation model, which mimics natural ZIKV vertical transmission [49].

In summary, ZIKV localizes to the ER for viral genome replication. These additional transcriptional and translational processes impart significant burden on the ER leading to ER stress and UPR induction. During the early stage of UPR, UPR effectors elicit adaptive responses to mitigate ER stress. These responses include the upregulation of chaperones and protein processing enzymes to promote and rectify protein folding, induction of ERAD to degrade misfolded/unfolded proteins, global translational arrest, and activation of autophagy and/or reticulophagy.

\section{ZIKV subverts other ER stress responses}

Beside UPR, ER stress also concomitantly induces several other cellular processes. Specifically, stress granules assembly and ER autophagy impart fundamental importance in regulating translational arrest and ER homeostasis, respectively. ZIKV have been reported to subvert these processes to allow the progression of viral replication.

\section{ZIKV inhibits cytoplasmic stress granules formation}

ER stress signal can be transmitted to other cellular components to rescue cell survival. A clear example to illustrate stress signal transmission is the generation of stress granules (SGs) in the cytoplasm to stall initiation of translation [70]. SGs assembly are generally triggered through two distinct categories of mechanisms: 1) eIF $2 \alpha$-independent and 2) eIF $2 \alpha$-dependent mechanisms [71]. In particular, the latter category, or more specifically eIF2 $\alpha$-phosphorylation, is mediated by PKR, PERK, general control non-derepressible-2 (GCN2), and heme-regulated inhibitor kinase (HRI) in response to diverse cellular stress signals [71]. SGs are ribonucleoprotein (RNP) structures primarily composed of non-translating mRNAs, stalled translation initiation complexes, and RNA-binding proteins [70]. The formation and components of SGs are reviewed in details here [71]. SGs assembly during global translational arrest negatively impact viral genome translation as the SGs reduce the accessibility of translational machinery complexes [72]. Following the relief of translation suppression, the SGs are disassembled via several mechanisms, one of which involves eIF $2 \alpha$ dephosphorylation by Growth Arrest and DNA-Damage-inducible 34 (GADD34) protein [73]. This allows the PIC to resume protein translation at the surface of the SGs, resulting in SGs shrinkage and disappearance [71].

Viruses have adopted several mechanisms to repress the assembly of stress granules and utilize SG protein components for viral polyprotein synthesis instead [74]. For example, herpes simplex virus genome encodes $\gamma_{1} 34.5$ protein that functionally mimics the activity of GADD34 and directs the dephosphorylation of eIF2 $\alpha$ [75]. Alternatively, coronaviruses repress the assembly of stress granules by asserting an inhibitory effect on PKR activation and upregulating the expression of GADD34 [76]. Likewise, ZIKV also suppresses the formation of stress granules in favor of virus replication by upregulating the expression of GADD34 [77]. Consistent with this finding, pharmacological inhibition of GADD34-mediated eIF2 $\alpha$ dephosphorylation rescued SGs assembly and decreased ZIKV particles production [77]. Additionally, another study reported that ZIKV proteins, namely capsid, NS3, NS2B-NS3, and NS4A proteins, suppressed SGs assembly; however, this modulation was observed in an eIF2 $\alpha$-independent manner [78]. ZIKV capsid inhibited SGs assembly by forming stable complexes with SG core proteins, Ras GTPase-activating proteinbinding protein 1 (G3BP1) and caprin-1, but not with T-cell-restricted intracellular antigen 1 related (TIAR) protein [78]. ZIKV utilized these SG core proteins for viral protein and virion production. Beside regulating eIF2 $\alpha$ phosphorylation and hijacking key SG proteins, RNA viruses were reportedly capable of interfering SGs assembly through cleaving and redistributing SGs nucleating factors [74]. It was previously reported that ZIKV NS2B-NS3 protease could cleave host antiviral factors to impair intrinsic host defense; intriguingly, ZIKV did not mediate the cleavage of SG factors even though SGs assembly was affected by ZIKV NS3 and NS2B-NS3 protease [77-81]. Nonetheless, ZIKV infection was found to facilitate the redistribution of TIAR to viral replication sites, which correlates to viral replication output $[77,78,81]$. Hence, it is unclear how ZIKV NS3, NS2B-NS3, and NS4A proteins block SGs formation and whether these viral proteins affect eIF $2 \alpha$ phosphorylation. Interestingly, a recent study also identified a key SG-interacting protein, human antigen R, exhibits substantial anti-ZIKV effect potentially by destabilizing the ZIKV RNA [81].

\section{ZIKV inhibits reticulophagy}

As previously discussed in Section 4, the induction of ER stress initiates several adaptive countermeasures such as upregulation of UPR pro-survival 
factors and ER enlargement to repair stress-induced damages and restore normal cellular functions. Interestingly, initial studies in mammalian and yeast cells revealed that a fraction of cells also contain autophagosome-like structures following ER stress induction $[82,83]$. These autophagic vacuoles expel selectively excised ER membrane containing aberrant protein aggregates [84]. This process, known as reticulophagy, is an additional mechanism that occurs in parallel with UPR to regulate ER volume and homeostasis [85]. Autophagy, including reticulophagy, is also a host innate defense mechanism as these autophagosomes have been shown to incorporate viral proteins for degradation [86].

Reticulophagy, also known as ER-phagy, is mediated by ER-phagy receptors such as Family with Sequence Similarity 134 Member B (FAM134B) and reticulon-3 (RTN3) proteins that reside on the ER membrane. These autophagy receptors sequester ER fragments via its LC3-interacting-region (LIR) domain interaction with autophagosomal-presenting microtubuleassociated protein 1 light chain 3 (MAP 1LC3) [87]. Similar to other ER-shaping proteins, FAM134B also possesses a reticulon homology domain (RHD), a motif that promotes membrane curvature [87].

In ZIKV-infected cells, depletion of FAM134B protein renders notable ER expansion and significant upregulation of ZIKV replication activity [80]. This is due to the proteolytic activity of ZIKV NS2B-NS3 that cleaves exposed RHD located on FAM134B protein, which impedes the oligomerization of FAM134B proteins and consequently, prevents ER membrane excision and reticulophagy from taking place [80]. This efficiently blocks host cell innate defense mechanism from degrading ZIKV proteins.

To summarize, ZIKV virus bypasses the UPR by inhibiting stress granules assembly and reticulophagy to ensure continuous viral protein translation and virion production while simultaneously protecting the virus from host cell defense mechanisms. Prolonged ER stress exacerbates the stress condition and leads to the activation of another arm of the UPR: cell death.

\section{ZIKV-induced ER stress leads to paraptosis-like cell death}

Countless studies showed that ZIKV infection causes cell death through several cell death mechanisms such as paraptosis and apoptosis $[12,88]$ Apoptotic events in ZIKV-infected cells occur through both the intrinsic and extrinsic pathways based on the activation of pathway-specific caspase-9 and caspase-8, respectively $[88,89]$. Interestingly, ZIKV Env protein is sufficiently capable to induce pro-apoptotic expression profile that represents intrinsic apoptosis including elevated expression of tumor protein p53, which correspondingly mediates the expression and activity of its downstream targets by suppressing anti-apoptotic BCL-2 and facilitating expression of pro-apoptotic Bcl-2associated X (BAX) [90]. This modulation consequently reduces mitochondrial membrane permeability; thus, aiding the release of cytochrome c, formation of apoptosome, and initiation of caspase cascade, which eventually leads to cell death [62]. Additionally, increased level of several apoptotic markers, such as tumor necrosis factor alpha and receptor-interacting protein 1, in ZIKV-positive microcephalic neural specimens are suggestive of extrinsic apoptotic activation [91].

As previously mentioned, ER stress elevated the expression of $C H O P$ to initiate apoptosis in ZIKVinfected cells $[48,49]$. Prolonged ER stress could also trigger non-apoptotic cell death as demonstrated by an investigation using time-lapse and electron microscopy that revealed the formation of extensive ERderived vacuoles within the cytoplasm of ZIKVinfected cells, which eventually resulted in paraptosislike death [28]. This observation is consistent with earlier report that showed cytoplasmic vacuolization in ZIKV-infected human skin biopsy specimens [13]. Vacuole formation was inhibited with class I PI3K/Akt inhibitor treatment. More importantly, pan-caspase inhibitor, ZVAD-FMK, only slightly rescued cell survival but did not affect vacuolization [28], implying that certain ZIKV strains, namely HD78788, PF13 and $\mathrm{NC14}$, induce cell death primarily through paraptosislike death. The role of PI3K/Akt pathway in ZIKVinduced cell death was verified by a recent study that reported treatment with AR-12, a celecoxib derivative kinase inhibitor, significantly inhibited the replication of ZIKV in A129 mice and improved mice survival mainly through Akt down-regulation [92].

\section{Conclusion}

$\mathrm{ZIKV}$, like other viruses, is an intracellular parasite that largely depends on the host biosynthetic, energetic and structural resources for successful viral replication and propagation. In particular, exploitation of these resources is manifested through the manipulation of the endoplasmic reticulum architecture and the processes that take place within or in proximity to the ER as summarized in Fig. 3. Briefly, ZIKV infection leads to structural changes of the ER such as ER enlargement due to the accumulation of misfolded/unfolded ZIKV proteins (Fig. 3d) and the formation of convoluted membranes, vesicle packets, zippered ER, and viroplasm-like structures for viral RNA replication (Fig. 3e). The accumulation of misfolded/unfolded proteins induce ER stress and triggers the UPR (Fig. 3f). In parallel, ER stress also initiate 


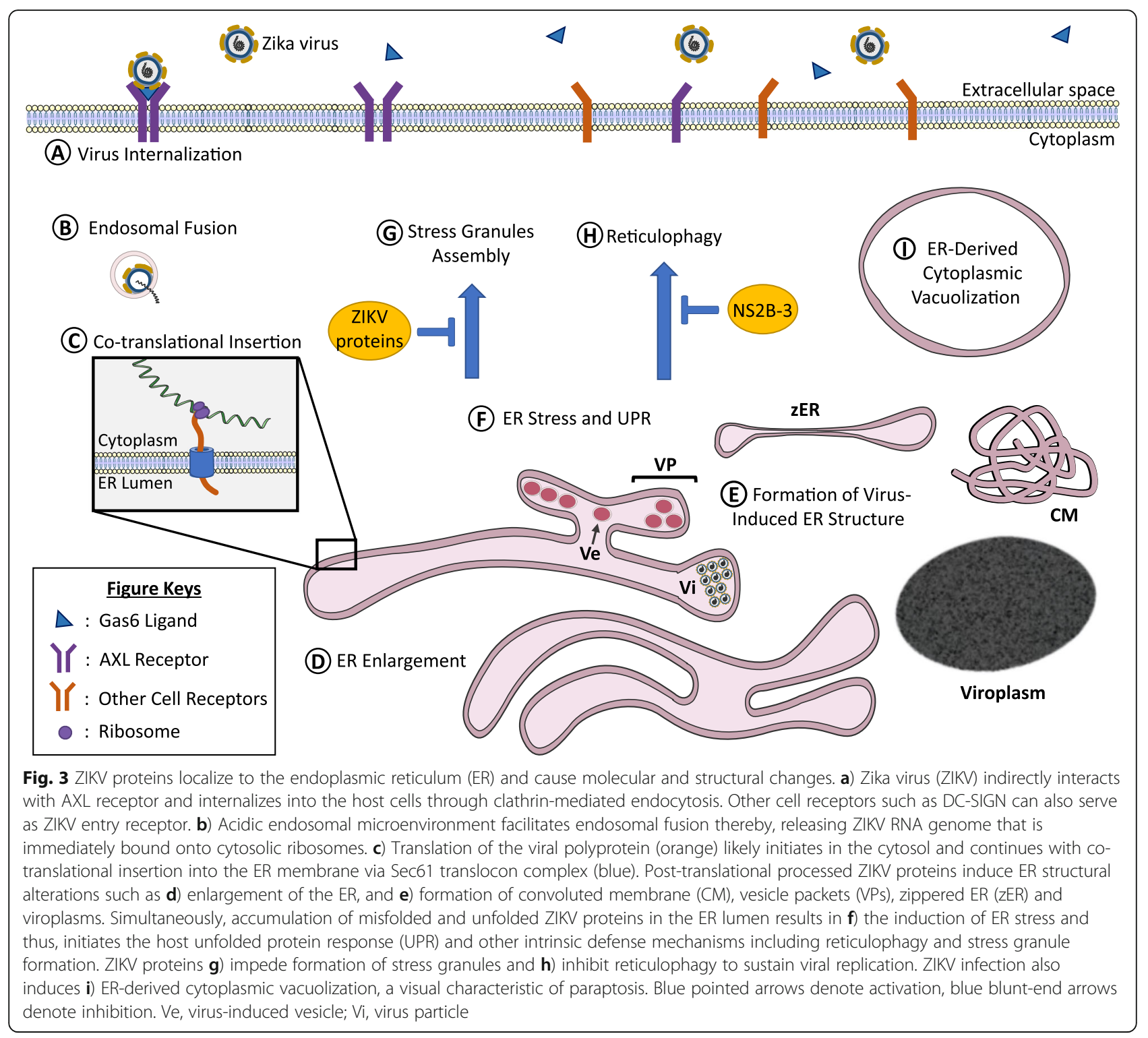

several response mechanisms such as stress granules assembly to impede global protein translation (Fig. 3g) and reticulophagy to remove damaged ER (Fig. 3h). However, ZIKV are able to bypass these processes through various strategies, which eventually lead to paraptosis-like cell death (Fig. 3i).

Although the number of ZIKV cases have subsided, ZIKV remains a significant threat due to the sporadic and unpredictable nature of its outbreak. In addition, ZIKV shares the same vectors with another widespread flavivirus, DENV [93], which is projected to increase exponentially in the future [94]. Thus, the potential re-occurrence of outbreaks, coupled with devastating neurological complications, warrants for extensive research to completely understand the virus pathophysiology for antiviral drug development. This is effectively demonstrated by several studies included in this review that employ multiple omics technologies to identify and target ER-associated ZIKV dependency factors and ER stress sensors. This strategy could pave the way in developing anti-ZIKV drugs.

\section{Abbreviations}

ATF6: Activating transcription factor 6; CM: Convoluted membrane; elF2: Eukaryotic initiation factor 2; EMC: ER membrane complex; ER: Endoplasmic reticulum; GRP78: Glucose-regulated protein 78; IRE1: Inositol-requiring enzyme 1; NS: Non-structural; PERK: Protein kinase RNA-activated (PKR)-like ER resident kinase; SGs: Stress granules; SPC: Signal peptidase complex; UPR: Unfolded protein response; VPs: Vesicle packets; XBP-1: x-box binding protein 1; zER: zippered ER; ZIKV: Zika virus

Acknowledgements

Not applicable. 


\section{Authors' contributions}

MIMR and ASK contributed to the topic conception and writing of the manuscript together. MIMR prepared the figures. MIMR, ASK, NNR and RY edited and revised the manuscript. All authors read and approved the final manuscript.

\section{Funding}

This work was supported by the grant [FRGS FP007-2017A] from the Ministry of Higher Education, Malaysia.

\section{Availability of data and materials}

Not applicable.

\section{Ethics approval and consent to participate}

Not applicable.

\section{Consent for publication}

Not applicable.

\section{Competing interests}

The authors declare that they have no competing interests.

\section{Received: 3 September 2019 Accepted: 14 January 2020}

\section{Published online: 20 January 2020}

\section{References}

1. Dick GW, Kitchen SF, Haddow AJ. Zika virus. I. Isolations and serological specificity. Trans R Soc Trop Med Hyg. 1952;46(5):509-20.

2. Musso D, Gubler DJ. Zika Virus. Clin Microbiol Rev. 2016;29(3):487-524.

3. Duffy MR, Chen TH, Hancock WT, Powers AM, Kool JL, Lanciotti RS, et al. Zika virus outbreak on Yap Island, Federated States of Micronesia. New Engl J Med. 2009;360(24):2536-43.

4. Oehler E, Watrin L, Larre P, Leparc-Goffart I, Lastere S, Valour F, et al. Zika virus infection complicated by Guillain-Barre syndrome - case report, French Polynesia, December 2013. Eurosurveillance. 2014;19(9):4-6.

5. Cao-Lormeau VM, Blake A, Mons S, Lastere S, Roche C, Vanhomwegen J, et al. Guillain-Barre syndrome outbreak associated with Zika virus infection in French Polynesia: a case-control study. Lancet. 2016;387(10027):1531-9.

6. Pan American Health Organization / World Health Organization. Epidemiological Alert: Increase of microcephaly in the northeast of Brazil. Washington D.C: PAHO / WHO; 2015.

7. Heymann DL, Hodgson A, Sall AA, Freedman DO, Staples JE, Althabe F, et al Zika virus and microcephaly: why is this situation a PHEIC? Lancet. 2016; 387(10020):719-21.

8. Rasmussen SA, Jamieson DJ, Honein MA, Petersen LR. Zika virus and birth defects - reviewing the evidence for causality. New Engl J Med. 2016; 374(20):1981-7.

9. Calvet G, Aguiar RS, Melo ASO, Sampaio SA, de Filippis I, Fabri A, et al. Detection and sequencing of Zika virus from amniotic fluid of fetuses with microcephaly in Brazil: a case study. Lancet Infect Dis. 2016;16(6):653-60.

10. Mlakar J, Korva M, Tul N, Popovic M, Poljsak-Prijatelj M, Mraz J, et al. Zika virus associated with microcephaly. New Engl J Med. 2016;374(10):951-8.

11. Garcez PP, Loiola EC, Da Costa RM, Higa LM, Trindade P, Delvecchio R, et al. Zika virus impairs growth in human neurospheres and brain organoids. Science. 2016;352(6287):816-8.

12. Li C, Xu D, Ye Q, Hong S, Jiang Y, Liu X, et al. Zika virus disrupts neural progenitor development and leads to microcephaly in mice. Cell Stem Cell. 2016;19(1):120-6.

13. Hamel R, Dejarnac O, Wichit S, Ekchariyawat P, Neyret A, Luplertlop N, et al. Biology of Zika virus infection in human skin cells. J Virol. 2015; 89(17):8880-96.

14. Meertens L, Labeau A, Dejarnac O, Cipriani S, Sinigaglia L, Bonnet-Madin L, et al. AxI mediates ZIKA virus entry in human glial cells and modulates innate immune responses. Cell Rep. 2017;18(2):324-33.

15. Persaud M, Martinez-Lopez A, Buffone C, Porcelli SA, Diaz-Griffero F. Infection by Zika viruses requires the transmembrane protein $A X L$, endocytosis and low pH. Virology. 2018;518:301-12.

16. Rawle RJ, Webster ER, Jelen M, Kasson PM, Boxer SG. pH dependence of Zika membrane fusion kinetics reveals an off-pathway state. Acs Central Sci. 2018;4(11):1503-10.
17. Sirohi D, Kuhn RJ. Zika virus structure, maturation, and receptors. J Infect Dis. 2017;216:S935-S44.

18. Sager G, Gabaglio S, Sztul E, Belov GA. Role of host cell secretory machinery in Zika virus life cycle. Viruses-Basel. 2018;10(10):559.

19. Hou WH, Cruz-Cosme R, Armstrong N, Obwolo LA, Wen FY, Hu WH, et al. Molecular cloning and characterization of the genes encoding the proteins of Zika virus. Gene. 2017;628:117-28.

20. Coyaud E, Ranadheera C, Cheng D, Goncalves J, Dyakov BJA, Laurent EMN, et al. Global Interactomics uncovers extensive Organellar targeting by Zika virus. Mol Cell Proteomics. 2018;17(11):2242-55.

21. Xing HP, Xu S, Jia FF, Yang Y, Xu CM, Qin CF, et al. Zika NS2B is a crucial factor recruiting NS3 to the ER and activating its protease activity. Virus Res. 2020:275:197793.

22. Grant A, Ponia SS, Tripathi S, Balasubramaniam V, Miorin L, Sourisseau M, et al. Zika virus targets human STAT2 to inhibit type I interferon signaling. Cell Host Microbe. 2016;19(6):882-90.

23. Xu S, Ci YL, Wang LJ, Yang Y, Zhang LL, Xu CM, et al. Zika virus NS3 is a canonical RNA helicase stimulated by NS5 RNA polymerase. Nucleic Acids Res. 2019:47(16):8693-707.

24. Zhang R, Miner JJ, Gorman MJ, Rausch K, Ramage H, White JP, et al. A CRISPR screen defines a signal peptide processing pathway required by flaviviruses. Nature. 2016;535(7610):164-8.

25. Savidis G, McDougall WM, Meraner P, Perreira JM, Portmann JM, Trincucci G, et al. Identification of Zika virus and dengue virus dependency factors using functional genomics. Cell Rep. 2016;16(1):232-46.

26. Lin DL, Inoue T, Chen YJ, Chang A, Tsai B, Tai AW. The ER membrane protein complex promotes biogenesis of dengue and Zika virus nonstructural multi-pass Transmembrane proteins to support infection. Cell Rep. 2019;27(6):1666-74.

27. Cox BD, Stanton RA, Schinazi RF. Predicting Zika virus structural biology: challenges and opportunities for intervention. Antivir Chem Chemother 2015;24(3-4):118-26.

28. Monel B, Compton AA, Bruel T, Amraoui S, Burlaud-Gaillard J, Roy N, et al. Zika virus induces massive cytoplasmic vacuolization and paraptosis-like death in infected cells. EMBO J. 2017;36(12):1653-68.

29. Scaturro P, Stukalov A, Haas DA, Cortese M, Draganova K, Plaszczyca A, et al. An orthogonal proteomic survey uncovers novel Zika virus host factors. Nature. 2018;561(7722):253-7.

30. Marceau CD, Puschnik AS, Majzoub K, Ooi YS, Brewer SM, Fuchs G, et al. Genetic dissection of Flaviviridae host factors through genome-scale CRISPR screens. Nature. 2016;535(7610):159-63.

31. Puschnik AS, Marceau CD, Ooi YS, Majzoub K, Rinis N, Contessa JN, et al. A small-molecule Oligosaccharyltransferase inhibitor with Pan-flaviviral activity. Cell Rep. 2017;21(11):3032-9.

32. Cortese M, Goellner S, Acosta EG, Neufeldt CJ, Oleksiuk O, Lampe M, et al. Ultrastructural characterization of Zika virus replication factories. Cell Rep. 2017:18(9):2113-23.

33. Romero-Brey I, Bartenschlager R. Endoplasmic reticulum: the favorite intracellular niche for viral replication and assembly. Viruses. 2016; 8(6):160.

34. Schwarz DS, Blower MD. The endoplasmic reticulum: structure, function and response to cellular signaling. Cell Mol Life Sci. 2016;73(1):79-94.

35. Offerdahl DK, Dorward DW, Hansen BT, Bloom ME. Cytoarchitecture of Zika virus infection in human neuroblastoma and Aedes albopictus cell lines. Virology. 2017;501:54-62

36. Rossignol ED, Peters KN, Connor JH, Bullitt E. Zika virus induced cellular remodelling. Cell Microbiol. 2017;19(8):e12740.

37. Liu J, Kline BA, Kenny TA, Smith DR, Soloveva V, Beitzel B, et al. A novel sheet-like virus particle array is a hallmark of Zika virus infection. Emerg Microbes Infec. 2018;7:69.

38. Aktepe TE, Liebscher S, Prier JE, Simmons CP, Mackenzie JM. The host protein Reticulon 3.1A is utilized by Flaviviruses to facilitate membrane Remodelling. Cell Rep. 2017;21(6):1639-54

39. Zhang XW, Xie XP, Xia HJ, Zou J, Huang LF, Popov VL, et al. Zika virus NS2Amediated Virion assembly. Mbio. 2019;10(5):e02375-19.

40. Maier HJ, Hawes PC, Cottam EM, Mantell J, Verkade P, Monaghan P, et al. Infectious bronchitis virus generates spherules from zippered endoplasmic reticulum membranes. Mbio. 2013:4(5):e00801-13.

41. Barreto-Vieira DF, Jacome FC, da Silva MAN, Caldas GC, de Filippis AMB, de Sequeira PC, et al. Structural investigation of C6/36 and Vero cell cultures infected with a Brazilian Zika virus. PLoS One. 2017;12(9):e0184397. 
42. Netherton $\mathrm{CL}$, Wileman $\mathrm{T}$. Virus factories, double membrane vesicles and viroplasm generated in animal cells. Curr Opin Virol. 2011;1(5):381-7.

43. Hoenen A, Liu WJ, Kochs G, Khromykh AA, Mackenzie JM. West Nile virus-induced cytoplasmic membrane structures provide partial protection against the interferon-induced antiviral MxA protein. J Gen Virol. 2007:88:3013-7.

44. Braakman I, Bulleid NJ. Protein folding and modification in the mammalian endoplasmic reticulum. Annu Rev Biochem. 2011:80:71-99.

45. Oakes SA, Papa FR. The role of endoplasmic reticulum stress in human pathology. Annu Rev Pathol-Mech. 2015;10:173-94.

46. Blondelguindi S, Cwirla SE, Dower WJ, Lipshutz RJ, Sprang SR, Sambrook JF, et al. Affinity panning of a library of peptides displayed on bacteriophages reveals the binding-specificity of Bip. Cell. 1993;75(4):717-28.

47. Turpin JFE.; Harrabi, W.; Kalamouni, C.E.; Desprès, P.; Krejbich-Trotot, P.; Viranaïcken, W. Crosstalk Between Endoplasmic Reticulum Stress and The Unfolded Protein Response During ZIKA Virus Infection. Preprint at https:// www.preprints.org/manuscript/201910.0024/v1 (2019).

48. Tan ZY, Zhang WP, Sun JH, Fu ZQ, Ke XL, Zheng CS, et al. ZIKV infection activates the IRE1-XBP1 and ATF6 pathways of unfolded protein response in neural cells. J Neuroinflamm. 2018;15:275.

49. Gladwyn-Ng I, Cordon-Barris L, Alfano C, Creppe C, Couderc T, Morelli G, et al. Stress-induced unfolded protein response contributes to Zika virusassociated microcephaly. Nat Neurosci. 2018;21(1):63-71.

50. Laguesse S, Creppe C, Nedialkova DD, Prevot PP, Borgs L, Huysseune S, et al. A dynamic unfolded protein response contributes to the control of cortical neurogenesis. Dev Cell. 2015;35(5):553-67.

51. Shen JS, Chen $X$, Hendershot L, Prywes R. ER stress regulation of ATF6 localization by dissociation of BiP/GRP78 binding and unmasking of golgi localization signals. Dev Cell. 2002;3(1):99-111.

52. Wang M, Wey SA, Zhang Y, Ye RS, Lee AS. Role of the unfolded protein response regulator GRP78/BiP in development, Cancer, and neurological disorders. Antioxid Redox Sign. 2009;11(9):2307-16.

53. Yoshida H, Matsui T, Yamamoto A, Okada T, Mori K. XBP1 mRNA is induced by ATF6 and spliced by IRE1 in response to ER stress to produce a highly active transcription factor. Cell. 2001;107(7):881-91.

54. Gardner BM, Walter P. Unfolded proteins are Ire1-activating ligands that directly induce the unfolded protein response. Science. 2011;333(6051): 1891-4.

55. Gardner BM, Pincus D, Gotthardt K, Gallagher CM, Walter P. Endoplasmic reticulum stress sensing in the unfolded protein response. Csh Perspect Biol. 2013;5(3):a013169.

56. Ali MMU, Bagratuni T, Davenport EL, Nowak PR, Silva-Santisteban MC, Hardcastle A, et al. Structure of the Ire1 autophosphorylation complex and implications for the unfolded protein response. EMBO J. 2011;30(5):894-905.

57. Prischi F, Nowak PR, Carrara M, Ali MMU. Phosphoregulation of Ire1 RNase splicing activity. Nat Commun. 2014;5:3554.

58. Zhang KZ, Kaufman RJ. From endoplasmic-reticulum stress to the inflammatory response. Nature. 2008;454(7203):455-62.

59. Sano R, Reed JC. ER stress-induced cell death mechanisms. Bba-Mol Cell Res. 2013;1833(12):3460-70

60. Wang $X Z$, Ron D. Stress-induced phosphorylation and activation of the transcription factor CHOP (GADD153) by p38 MAP kinase. Science. 1996; 272(5266):1347-9.

61. Sari FR, Widyantoro B, Thandavarayan RA, Harima M, Lakshmanan AP, Zhang $\mathrm{S}$, et al. Attenuation of CHOP-mediated myocardial apoptosis in pressureoverloaded dominant negative p38alpha mitogen-activated protein kinase mice. Cell Physiol Biochem. 2011;27(5):487-96.

62. Hu H, Tian MX, Ding C, Yu SQ. The C/EBP homologous protein (CHOP) transcription factor functions in endoplasmic reticulum stress-induced apoptosis and microbial infection. Front Immunol. 2019;9:3083.

63. Ubeda M, Wang XZ, Zinszner H, Wu I, Habener JF, Ron D. Stress-induced binding of the transcriptional factor $\mathrm{CHOP}$ to a novel DNA control element. Mol Cell Biol. 1996;16(4):1479-89.

64. Puthalakath H, O'Reilly LA, Gunn P, Lee L, Kelly PN, Huntington ND, et al. ER stress triggers apoptosis by activating BH3-only protein Bim. Cell. 2007; 129(7):1337-49.

65. Cormier JH, Tamura T, Sunryd JC, Hebert DN. EDEM1 recognition and delivery of Misfolded proteins to the SEL1L-containing ERAD complex. Mo Cell. 2009;34(5):627-33.

66. Shaffer AL, Shapiro-Shelef M, Iwakoshi NN, Lee AH, Qian SB, Zhao H, et al. XBP1, downstream of Blimp-1, expands the secretory apparatus and other organelles, and increases protein synthesis in plasma cell differentiation. Immunity. 2004;21(1):81-93.

67. Schuck S, Prinz WA, Thorn KS, Voss C, Walter P. Membrane expansion alleviates endoplasmic reticulum stress independently of the unfolded protein response. J Cell Biol. 2009;187(4):525-36.

68. Wang P, Li JZ, Tao JH, Sha BD. The luminal domain of the ER stress sensor protein PERK binds misfolded proteins and thereby triggers PERK oligomerization. J Biol Chem. 2018;293(11):4110-21.

69. Holcik M, Sonenberg N. Translational control in stress and apoptosis. Nat Rev Mol Cell Bio. 2005;6(4):318-27.

70. Buchan JR, Parker R. Eukaryotic stress granules: the ins and outs of translation. Mol Cell. 2009;36(6):932-41.

71. Panas MD, Ivanov P, Anderson P. Mechanistic insights into mammalian stress granule dynamics. J Cell Biol. 2016;215(3):313-23.

72. McCormick C, Khaperskyy DA. Translation inhibition and stress granules in the antiviral immune response. Nat Rev Immunol. 2017;17(10):647-60.

73. Brush MH, Weiser DC, Shenolikar S. Growth arrest and DNA damageinducible protein GADD34 targets protein phosphatase 1 alpha to the endoplasmic reticulum and promotes dephosphorylation of the alpha subunit of eukaryotic translation initiation factor 2. Mol Cell Biol. 2003;23(4): 1292-303.

74. Zhang Q, Sharma NR, Zheng ZM, Chen MZ. Viral regulation of RNA granules in infected cells. Virol Sin. 2019:34(2):175-91.

75. He B, Gross M, Roizman B. The gamma(1)34.5 protein of herpes simplex virus I complexes with protein phosphatase 1 alpha to dephosphorylate the alpha subunit of the eukaryotic translation initiation factor 2 and preclude the shutoff of protein synthesis by double-stranded RNA-activated protein kinase. P Natl Acad Sci USA. 1997;94(3):843-8.

76. Wang XX, Liao Y, Yap PL, Png KJ, Tam JP, Liu DX. Inhibition of protein kinase $R$ activation and Upregulation of GADD34 expression play a synergistic role in facilitating coronavirus replication by maintaining De novo protein synthesis in virus-infected cells. J Virol. 2009:83(23):12462-72.

77. Amorim R, Temzi A, Griffin BD, Mouland AJ. Zika virus inhibits elF2 alphadependent stress granule assembly. Plos Neglect Trop D. 2017;11(7):e0005775.

78. Hou SM, Kumar A, Xu ZK, Airo AM, Stryapunina I, Wong CP, et al. Zika virus hijacks stress granule proteins and modulates the host stress response. J Virol. 2017;91(16):e00474-17.

79. Ding Q, Gaska JM, Douam F, Wei L, Kim D, Balev M, et al. Species-specific disruption of STING-dependent antiviral cellular defenses by the Zika virus NS2B3 protease. P Natl Acad Sci USA. 2018;115(27):E6310-E8.

80. Lennemann NJ, Coyne CB. Dengue and Zika viruses subvert reticulophagy by NS2B3-mediated cleavage of FAM134B. Autophagy. 2017;13(2):322-32.

81. Bonenfant G, Williams N, Netzband R, Schwarz MC, Evans MJ, Pager CT. Zika virus subverts stress granules to promote and restrict viral gene expression. J Virol. 2019;93(12):e00520-19.

82. Bernales S, McDonald KL, Walter P. Autophagy counterbalances endoplasmic reticulum expansion during the unfolded protein response. PLoS Biol. 2006;4(12):2311-24.

83. Ding WX, Ni HM, Gao WT, Hou YF, Melan MA, Chen XY, et al. Differential effects of endoplasmic reticulum stress-induced autophagy on cell survival. J Biol Chem. 2007;282(7):4702-10.

84. Teckman $\mathrm{JH}$, Perlmutter $\mathrm{DH}$. Retention of mutant alpha(1)-antitrypsin Z in endoplasmic reticulum is associated with an autophagic response. Am J Physiol-Gastr L. 2000;279(5):G961-G74.

85. Schuck S, Gallagher CM, Walter P. ER-phagy mediates selective degradation of endoplasmic reticulum independently of the core autophagy machinery. J Cell Sci. 2014;127(18):4078-88.

86. Levine B, Mizushima N, Virgin HW. Autophagy in immunity and inflammation. Nature. 2011;469(7330):323-35.

87. Khaminets $A$, Heinrich $T$, Mari M, Grumati $P$, Huebner AK, Akutsu M, et al. Regulation of endoplasmic reticulum turnover by selective autophagy. Nature. 2015:522(7556):354-8.

88. Souza BSF, Sampaio GLA, Pereira CS, Campos GS, Sardi SI, Freitas LAR, et al. Zika virus infection induces mitosis abnormalities and apoptotic cell death of human neural progenitor cells. Sci Rep-Uk. 2016;6:39775.

89. Hastings AK, Hastings K, Uraki R, Hwang J, Gaitsch N, Dhaliwal K, et al. Loss of the TAM receptor Axl ameliorates severe Zika virus pathogenesis and reduces apoptosis in microglia. Iscience. 2019:13:339-50.

90. Liu J, Li QQ, Li XX, Qiu ZZ, Li A, Liang WH, et al. Zika virus envelope protein induces G2/M cell cycle arrest and apoptosis via an intrinsic cell death signaling pathway in neuroendocrine PC12 cells. Int J Biol Sci. 2018;14(9):1099-108. 
91. de Sousa JR, Azevedo RSS, Martins AJ, Araujo MTF, Moutinho ERC, Vasconcelos BCB, et al. Correlation between apoptosis and in situ immune response in fatal cases of microcephaly caused by Zika virus. Am J Pathol. 2018:188(11):2644-52.

92. Chan JFW, Zhu Z, Chu H, Yuan SF, Chik KKH, Chan CCS, et al. The celecoxib derivative kinase inhibitor AR-12 (OSU-03012) inhibits Zika virus via downregulation of the PI3K/Akt pathway and protects Zika virus infected A129 mice: a host-targeting treatment strategy. Antivir Res. 2018;160:38-47.

93. Boyer S, Calvez E, Chouin-Carneiro T, Diallo D, Failloux AB. An overview of mosquito vectors of Zika virus. Microbes Infect. 2018;20(11-12):646-60.

94. Messina JP, Brady OJ, Golding N, Kraemer MUG, Wint GRW, Ray SE, et al. The current and future global distribution and population at risk of dengue. Nat Microbiol. 2019;4(9):1508-15.

\section{Publisher's Note}

Springer Nature remains neutral with regard to jurisdictional claims in published maps and institutional affiliations.

Ready to submit your research? Choose BMC and benefit from:

- fast, convenient online submission

- thorough peer review by experienced researchers in your field

- rapid publication on acceptance

- support for research data, including large and complex data types

- gold Open Access which fosters wider collaboration and increased citations

- maximum visibility for your research: over $100 \mathrm{M}$ website views per year

At BMC, research is always in progress.

Learn more biomedcentral.com/submissions 